\title{
Evidence of increased Cathepsin B/L and decreased TMPRSS2 usage for cell entry by the SARS-CoV-2 Omicron variant
}

5 Pranesh Padmanabhan ${ }^{1, *}$, Narendra M. Dixit ${ }^{2,3, *}$

6

$7{ }^{1}$ Clem Jones Centre for Ageing Dementia Research, Queensland Brain Institute, The

8 University of Queensland, Brisbane, Australia 4072

$9 \quad{ }^{2}$ Department of Chemical Engineering, Indian Institute of Science, Bangalore, India 560012

$10{ }^{3}$ Centre for Biosystems Science and Engineering, Indian Institute of Science, Bangalore, India $11 \quad 560012$

13 *Correspondence:

14 Pranesh Padmanabhan, Narendra M. Dixit

15 Email: p.padmanabhan@uq.edu.au; narendra@iisc.ac.in

17 Letter details:

18 Main Text: 1200 words; Figures: 1; References: 10 
The SARS-CoV-2 Omicron variant appears to exhibit altered cell tropism and entry properties compared to other variants. Here, analysing recent data, we found a strong positive correlation between the entry efficiency of the Omicron variant and the relative usage of the host protease Cathepsin B/L by the original SARS-CoV-2 strain for cell entry. We developed a mathematical model to quantify entry efficiency in in vitro assays and found that the Omicron variant displayed $>4$-fold improved efficiency in using Cathepsin B/L for entry in 293T-ACE2 cells compared to the original strain. The preferential usage of Cathepsin B/L over TMPRSS2 for entry may explain the altered cell tropism of the Omicron variant and have implications for our understanding of its infectivity and transmissibility as well as for interventions. $25^{\text {th }}$ November 2021, is fast becoming the dominant strain in several countries. It harbours as many as 37 mutations in the spike protein compared to the original SARS-CoV-2 strain [1] and is able to evade neutralising antibodies generated by previously infected or vaccinated individuals [1-3], possibly leading to its increased transmissibility and rapid global spread. Emerging data indicates that the high number of mutations in the Omicron variant affects its viral entry properties and cell tropism [2-5], which in turn may influence its ability to establish infection post-exposure and the severity of the subsequent symptoms. In this letter, we analyse this new data and find that the Omicron variant has substantially altered preferences for cell entry pathways compared to the earlier variants. Further, using a mathematical model, we quantify the extent of the differential usage of the entry pathways by the Omicron variant. 
tropism may be altered and its entry efficiency different from the original and other variants in a cell line-dependent manner [2-5]. For instance, SARS-CoV-2 pseudotyped virus bearing either the B.1 or the Delta variant spike protein showed higher entry efficiency than the Omicron pseudotyped virus in Caco-2 (human, colon) and Calu-3 (human, lung) cells, whereas the Omicron pseudotyped virus entered Vero (African green monkey, kidney) and 293T (human, kidney) cells more efficiently than the other variants [3]. Similar trends were observed in live SARS-CoV-2 virus infection assays $[4,5]$; the Delta variant infection spread was significantly greater than the Omicron variant in Calu-3 cells [4, 5], whereas the spread of the two variants was similar in VeroE6 cells [4]. What causes the relative efficiency of Omicron virus entry to be higher in some cells and lower in others than other variants?

The first step in SARS-CoV-2 entry into target cells is the binding of the viral spike protein, S, with the host cell surface receptor angiotensin-converting enzyme 2 (ACE2). Cell tropism is thus expected to be affected by ACE2 expression levels [6-8]. The Omicron spike protein binds soluble human ACE2 strongly [1, 3]. Further, ACE2 is necessary for the entry of all SARS-CoV-2 variants [2-6]. Thus, any variation in ACE2 expression across cell types is likely to have similar effects on the entry efficiencies of all the variants. The cell typedependent variation in the entry efficiency of the Omicron variant is therefore unlikely to arise from the variations in the ACE2 expression level across cell types. Omicron spike protein incorporation into pseudotyped virus appears to be compromised compared to the Delta and Wuhan D614G strains [5]. However, this reduction in spike protein density is likely to decrease the Omicron entry efficiency across all cell types, thus ruling it out as a potential cause of the differential entry efficiency observed.

Following ACE2 engagement, successful entry requires that the spike protein be cleaved into subunits by host proteases, either by the transmembrane serine protease TMPRSS2 at the plasma membrane or the cysteine proteases Cathepsin B and Cathepsin L in the 
endosomal vesicles. The proteases TMPRSS2 and Cathepsin $\mathrm{B} / \mathrm{L}$ are thought to work independently, facilitating SARS-CoV-2 entry through two independent pathways [8,9] (Fig. 1A). We recently analysed SARS-CoV-2 pseudotyped virus infection of several cell lines [6] using a mathematical model of SARS-CoV-2 entry and found that the relative usage of TMPRSS2 and Cathepsin B/L entry pathways by the original SARS-CoV-2 strain varied widely across cell lines [9]. For example, Vero cells predominantly admitted entry through the Cathepsin B/L pathway, whereas Calu-3 cells allowed entry via the TMPRSS2 pathway. Vero cells overexpressing TMPRSS2 permitted entry via both pathways. Importantly, the original strain displayed the ability to use either pathway, with the preferred pathway possibly based on the relative expression levels of the two proteases [8]. We reasoned that the Omicron variant might use these pathways differently from the original (or the Delta) strain, possibly underlying its different cell tropism.

To test this, we extended the above analysis to the recent experiments of Hoffmann et al. [3] and found a correlation between the Omicron virus entry efficiency and the relative usage of the two entry pathways by the original strain (Fig. 1B). With cell lines (Calu-3 and Caco-2) where the usage of the TMPRSS2 entry pathway by the original strain was dominant, the Omicron pseudotyped virus entry was significantly less efficient than the B.1 and delta strains. By contrast, the Omicron virus entry was more efficient in cell lines (Calu-3 and Caco2) where the Cathepsin B/L entry pathway usage by the original strain was dominant (Fig. 1B). One possible interpretation of this relationship is that the Omicron variant entry is relatively less effective through the TMPRSS2 pathway and more effective via the Cathepsin $\mathrm{B} / \mathrm{L}$ pathway than the other strains. Consistent with this notion, camostat mesylate, a TMPRSS2 inhibitor, was less potent against the Omicron variant than the Delta variant in blocking live virus infection of VeroE6 cells overexpressing TMPRSS2, which allows entry via both pathways [4]. Moreover, syncytium formation in a cell-cell fusion assay, which requires 
TMPRSS2 but not Cathepsins, was severely impaired for the Omicron variant compared to both the Delta [4, 5] and the Wuhan-Hu-1 D614G [5] strains. The increased efficiency of Cathepsin B/L usage together with the reduced efficiency of TMPRSS2 usage by the Omicron variant may explain its altered cell tropism relative to the original strain or other variants.

To quantify the increased efficiency of the Cathepsin B/L pathway usage by the Omicron variant, we developed a mathematical model and applied it to analyse data from Garcia-Beltran et al. [2], who measured the fraction of 293T-ACE2 cells infected by pseudotyped virus bearing the wild-type or the Omicron spike proteins at different viral concentrations (Fig. 1C). We derived an analytical expression to describe infections in the pseudotyped virus infection assay (Supplementary Information, Text S1). Accordingly, the

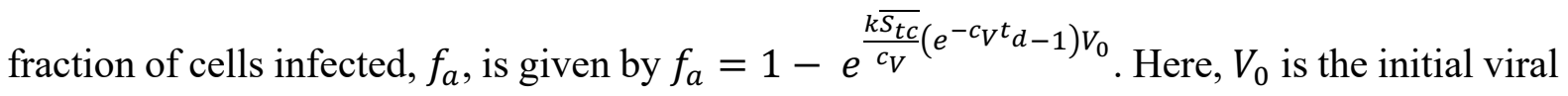
concentration, and $\overline{S_{t c}}$ is the mean susceptibility of the cell population to virus entry, accounting for the usage of both the pathways. (See Text S1 for the description of other model parameters.) Because 293T-ACE2 cells predominantly permit original strain entry via the Cathepsin B/L pathway [9] and given the relatively poor efficiency of TMPRSS2 usage by the Omicron variant (Fig. 1B), we let $\overline{S_{t c}}=\overline{S_{c}}$, where $\overline{S_{c}}$ is the mean susceptibility through the Cathepsin B/L pathway. Our model provided good fits to the data (Fig. 1C) and estimated 4.6fold increased usage of the Cathepsin pathway by the Omicron variant in 293T-ACE2 cells relative to the original strain (more details in Text $\mathrm{S} 1$ ).

The switch in the Omicron variant's preference for entry pathways may have clinical implications. Drugs targeting TMPRSS2, such as camostat mesylate [6], and Cathepsin B/L pathway, such as hydroxychloroquine [10], are in clinical development. The effectiveness of TMPRSS2 inhibitors is likely to decrease and that of Cathepsin $\mathrm{B} / \mathrm{L}$ inhibitors is likely to increase against the Omicron strain. Furthermore, our mathematical model previously predicted synergy between TMPRSS2 and Cathepsin B/L inhibitors against SARS-CoV-2 
bioRxiv preprint doi: https://doi.org/10.1101/2022.01.13.476267; this version posted January $18,2022$. The copyright holder for this preprint (which was not certified by peer review) is the author/funder, who has granted bioRxiv a license to display the preprint in perpetuity. It is made available under aCC-BY-NC-ND 4.0 International license.

infection [9]. Because the Omicron variant's preference for the TMPRSS2 pathway is reduced but not eliminated, combination treatment with TMPRSS2 and Cathepsin B/L inhibitors may exhibit limited but possibly useful synergy against Omicron virus infection of cells that admit both entry pathways. Future studies may establish these implications quantitatively. We suggest that pseudotyped virus infection experiments at different viral concentrations together with inhibitors of TMPRSS2 and Cathepsin B/L pathways, analysed as described here and in Padmanabhan et al. [9], might help quantify the relative TMPRSS2 and Cathepsin B/L pathway usage by different strains across cell types including Omicron, potentially explaining their cell tropism and the efficacy of the above treatments.

In summary, our analyses support the notion that the Omicron variant has shifted to better usage of the Cathepsin B/L entry pathway at the expense of the TMPRSS2 pathway. It would be interesting to see how these changes in the entry pathway usage observed in in vitro conditions relate to disease severity and transmissibility in animal models and humans.

\section{ACKNOWLEDGEMENTS}

We thank Rajat Desikan for help with Figure 1A.

\section{COMPETING INTERESTS}

The authors declare that no conflicts of interests exist.

\section{REFERENCES}

1. Cameroni E, Bowen JE, Rosen LE, Saliba C, Zepeda SK, Kulap K, et al. Broadly neutralizing antibodies overcome SARS-CoV-2 Omicron antigenic shift. Nature. 2021.

2. Garcia-Beltran WF, Denis KJS, Hoelzemer A, Lam EC, Nitido AD, Sheehan ML, et al. mRNA-based COVID-19 vaccine boosters induce neutralizing immunity against SARS-CoV2 Omicron variant. Cell. 2021. 
146 3. Hoffmann $M$, et al. The Omicron variant is highly resistant against antibody-mediated neutralization - implications for control of the COVID-19 pandemic. 2021.

148 4. Zhao H, Lu L, Peng Z, Chen LL, Meng X, Zhang C, et al. SARS-CoV-2 Omicron variant 149 shows less efficient replication and fusion activity when compared with delta variant in 150 TMPRSS2-expressed cells. Emerg Microbes Infect. 2021;1-18.

151 5. Meng B, Ferreira IATM, Abdullahi A, Saito A, Kimura I, al. e. SARS-CoV-2 Omicron spike mediated immune escape, infectivity and cell-cell fusion. bioRxiv. 2021.

153 6. Hoffmann M, Kleine-Weber H, Schroeder S, Krüger N, Herrler T, Erichsen S, et al. SARS154 CoV-2 cell entry depends on ACE2 and TMPRSS2 and is blocked by a clinically proven 155 protease inhibitor. Cell. 2020;181:271-80.e8.

156 7. Liu J, Li Y, Liu Q, Yao Q, Wang X, Zhang H, et al. SARS-CoV-2 cell tropism and 157 multiorgan infection. Cell Discov. 2021;7:17.

158 8. Koch J, Uckeley ZM, Doldan P, Stanifer M, Boulant S, Lozach PY. TMPRSS2 expression 159 dictates the entry route used by SARS-CoV-2 to infect host cells. EMBO J. 2021;40:e107821.

160 9. Padmanabhan P, Desikan R, Dixit NM. Targeting TMPRSS2 and Cathepsin B/L together 161 may be synergistic against SARS-CoV-2 infection. PLoS Comput Biol. 2020;16:e1008461.

162 10. Ou T, Mou H, Zhang L, Ojha A, Choe H, Farzan M. Hydroxychloroquine-mediated inhibition of SARS-CoV-2 entry is attenuated by TMPRSS2. PLoS Pathog. 2021;17:e1009212. 
A

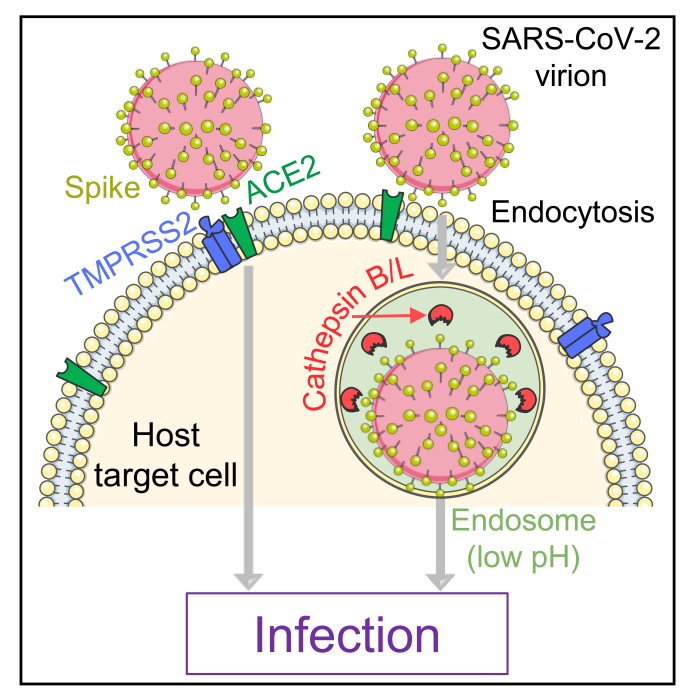

C

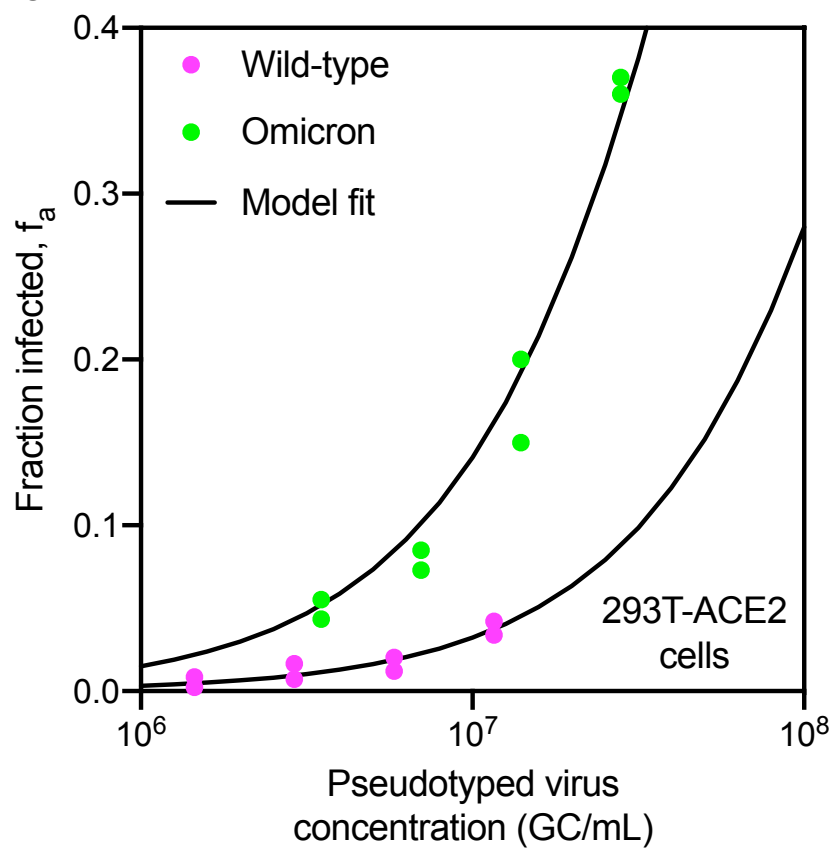

B
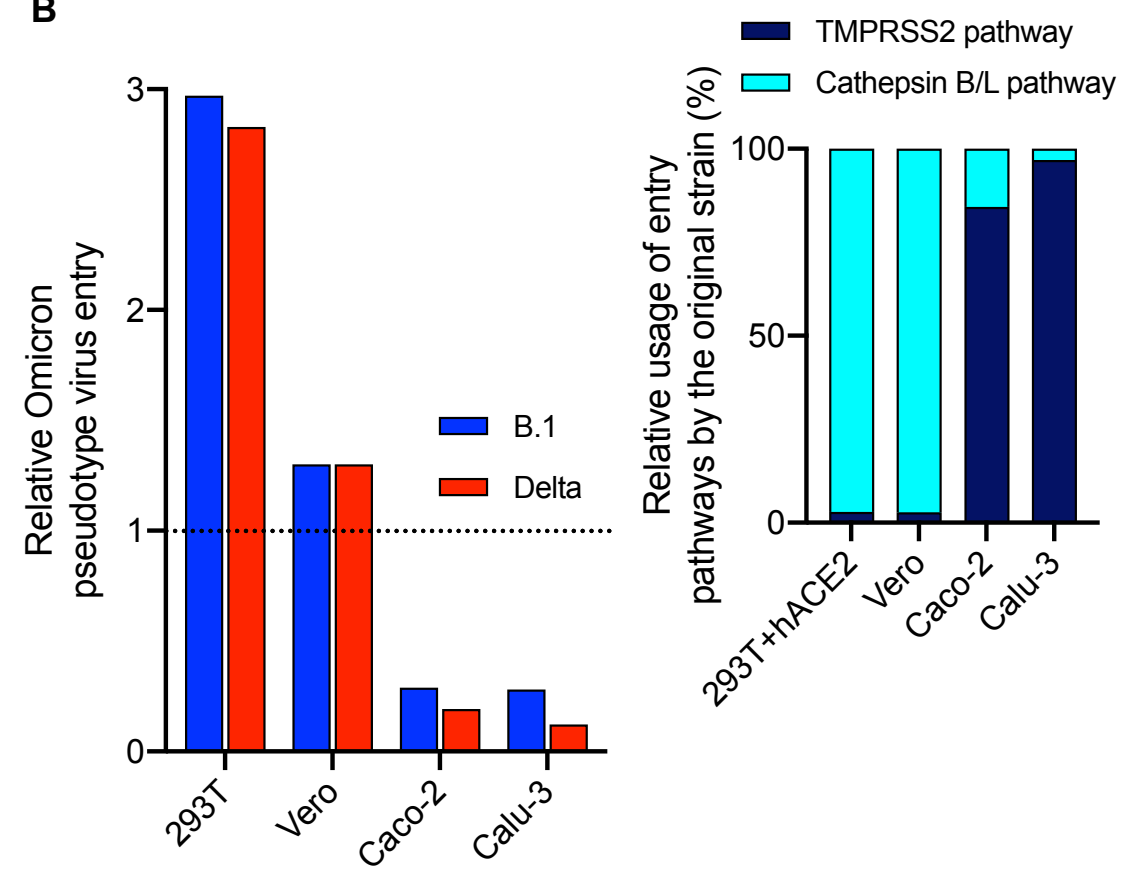

Figure 1. Omicron variant entry efficiency relative to B.1 and Delta strains depends on independent entry pathways accessible to SARS-CoV-2. (B) The Omicron entry efficiency relative to either the B.1 (blue) or the Delta (red) variant across cell types. Data was taken from

170 Hoffmann et al. [3]. Inset, the relative usage of the entry pathways by the original strain. Data 
172 experimental data (filled circles) taken from Garcia-Beltran et al. [2] measuring the fraction of

173 cells infected by pseudotyped virus bearing either the wild-type or the Omicron spike proteins.

174 In B and C, experimental data was extracted using Engauge Digitizer 12.1. In C, Data was fit

175 using the tool NLINFIT in MATLAB R2017b. 